\title{
Hot topic: Detecting digital dermatitis with computer vision
}

 \\ ${ }^{1}$ Department of Medical Sciences, School of Veterinary Medicine, University of Wisconsin, Madison 53706 \\ ${ }^{2}$ Department of Pathobiological Sciences, School of Veterinary Medicine, University of Wisconsin, Madison 53706 \\ ${ }^{3}$ Department of Computer Sciences, University of Wisconsin, Madison 53706
}

\begin{abstract}
Digital dermatitis (DD) is linked to severe lameness, infertility, and decreased milk production in cattle. Early detection of DD provides an improved prognosis for treatment and recovery; however, this is extremely challenging on commercial dairy farms. Computer vision (COMV) models can help facilitate early DD detection on commercial dairy farms. The aim of this study was to develop and implement a novel COMV tool to identify DD lesions on a commercial dairy farm. Using a database of more than 3,500 DD lesion images, a model was trained using the YOLOv2 architecture to detect the M-stages of DD. The YOLOv2 COMV model detected DD with an accuracy of $71 \%$, and the agreement was quantified as "moderate" by Cohen's kappa when compared with a human evaluator for the internal validation. In the external validation, the YOLOv2 COMV model detected DD with an accuracy of $88 \%$ and agreement was quantified as "fair" by Cohen's kappa. Implementation of COMV tools for DD detection provides an opportunity to identify cows for DD treatment, which has the potential to lower DD prevalence and improve animal welfare on commercial dairy farms.
\end{abstract}

Key words: Computer vision, digital dermatitis, machine learning

\section{Hot Topic}

First discovered in 1974 by Cheli and Mortellaro, digital dermatitis (DD) is a painful superficial ulceration along the plantar-palmar interdigital space of the hoof (Döpfer, 2009). Linked to decreased milk production, severe lameness, and infertility, DD is a major animal welfare and production problem in dairy

Received August 20, 2019.

Accepted April 27, 2020.

*Corresponding author: pcernek@wisc.edu cattle (Vanhoudt et al., 2019). Digital dermatitis has been estimated to affect up to $75 \%$ of dairy herds in the United States (Cramer et al., 2009; Krull et al., 2016) and can reach $>70 \%$ prevalence within herds (Jacobs et al., 2019; Zinpro Inc., 2019). Prompt identification and treatment of lesions reduces prevalence within a herd (Schulz et al., 2016), but requires extensive employee training in an industry with a high employee turnover rate (Moore, 2012). Consequently, DD detection is often suboptimal on commercial dairy farms.

Computer vision (COMV) methods for disease detection, such as those used for facial and fingerprint recognition on hand-held devices, are becoming more common in veterinary medicine (Chollet, 2018; Sandle, 2018) and provide a unique opportunity for DD detection. Even though early detection of DD is difficult, it is beneficial for animal welfare and productivity because early detection leads to prompt treatment. Furthermore, continuously scoring lesions allows for optimized treatment plans that were previously unattainable. Computer vision could be used to improve early DD detection on commercial dairy farms.

In this project, novel COMV tools were developed and implemented on a commercial dairy farm to provide early detection of the M-stages (DD lesion classification system; Döpfer et al., 1997; Berry et al., 2012). The COMV approach, YOLOv2 (named from the phrase "You Only Look Once"), uses convolutional neural networks to process the global context of images, and enables detection speeds of up to 155 frames per second (Redmon et al., 2015). The high speed of detection and accuracy makes YOLOv2 desirable for real-time object detection (Gutierre, 2018). With realtime object detection as a high priority in this project, we selected an approach based on YOLOv2.

This study aimed to (1) train COMV models using transfer learning with YOLOv2 and a recommended database of at least 1,000 images of each DD M-stage (Chollet, 2016), (2) implement the model in an external validation trial on a commercial dairy farm, and (3) quantify agreement between COMV model predic- 
tions and a trained investigator using accuracy and Cohen's kappa (Viera and Garrett, 2005).

Dairy cows affected by DD were selected from 2 commercial dairy farms in the midwestern United States. We directed GoPro Hero 5 Black cameras (GoPro Inc., San Mateo, CA) at the interdigital space of cows' hind feet to collect MP4 video recordings. Screenshot JPGs $(2,510)$ were collected from the MP4 video recordings. Additionally, JPG images (681) from traditional and cell phone cameras were obtained when researchers, hoof trimmers, and veterinarians encountered DD hooves in other studies in Wisconsin; Manitoba, Canada; and Utrecht, the Netherlands. These 681 JPGs were placed into the image database. Utilizing both JPG and MP4 screenshot JPG formats created necessary diversity of the image files. The images and videos were collected in a variety of settings and scenarios to produce a diverse database of images similar to real life situations. We obtained 467 images when hooves were lifted in trimming chutes, and the remaining images $(2,724)$ were collected randomly as cattle stood on a flat surface in the cattle housing area, in an automated milking system, or passed by a stationary camera mounted at foot level in a rotary milking parlor. All images were collected with the camera facing the rear foot with a clear view of the interdigital space of the hoof. In this way, an image database was created that was representative of DD in commercial and typical dairy farms. Image collection occurred between June 2018 and March 2019.

All images $(3,191)$ in the database were scored for M-stages of DD by a trained investigator using the M-stage DD classification system described by Döpfer et al. (1997) and Berry et al. (2012). Based on this classification system, cattle images in which normal skin was observed on the foot were classified as M0 (healthy claws). Images that showed acute, active ulcerative DD lesions $\geq 20 \mathrm{~mm}$ in diameter were classified as M2, and images that showed chronic lesions characterized by a thickened epithelium (hyperkeratosis) were classified as $\mathbf{M} 4 \mathbf{H}$ (chronically affected claws). For the purpose of creating a COMV model, M0 images were grouped together with $\mathrm{M} 4 \mathrm{H}$ images because the purpose of the COMV detection model was to identify M2 lesions from all other lesions. The combined $\mathrm{M} 0$ and $\mathrm{M} 4 \mathrm{H}$ images were contrasted against M2. No attempt was made to detect M0 and $\mathrm{M} 4 \mathrm{H}$ separately because, at the image resolution used in this study, the visual difference between the 2 lesions was minimal.

Due to an unequal number of images per M-stage, JPG images were augmented in Python version 3.6 (python.org) through Anaconda (Anaconda Inc., Austin, TX) with Augmentor version 0.2.6 (pypi. org). Augmentation is a common procedure for creating new training images similar to the original by mirroring, rotating, flipping, shifting, or shearing the images (Chollet, 2018). The M4H image database was augmented from $50 \mathrm{JPG}$ images to 500 images. The 241 M0 JPG images and the 390 M2 JPG images were not augmented. Screenshot images from MP4 video recordings were not augmented due to the lower quality of these images, which have a decreased capacity for acceptable augmentation. After augmentation, the image database included 1,177 M0 images (936 screenshot JPG images and 241 JPG images), 1,414 M4H images (914 screenshot JPG images and 500 JPG images), and 1,050 M2 images (660 screenshot JPG images and 390 JPG images).

After the M0/M4H and M2 image database was compiled, matplotlib (matplotlib.org), OpenCV version 4.1.0 (opencv.org), and NumPy v1.16.3 (numpy. org) were used to rename images and to generate xml annotations in Python. The YOLOv2 COMV model was trained to differentiate between 2 DD image classifications: $\mathrm{M} 0 / \mathrm{M} 4 \mathrm{H}$ and $\mathrm{M} 2$ lesions using 2,591 M0/M4H images and 1,050 M2 images. We used the darknet framework (Redmon, 2013) in Windows 10 (Microsoft Corp., Redmond, WA) with a batch size of 16 and a learning rate of 0.00001 for 1,000 epochs supported by an NVIDIA GeForce 1070 Ti graphics processing unit (NVIDIA Corporation, Santa Clara, CA), TensorFlow version 1.3.0 (tensorflow.org), and CUDA version 8 and cuDNN version 7.1.3 (developer. nvidia.com) to perform the training of the YOLOv2 model. Training metrics were graphed in TensorBoard version 1.12.0. The above hardware was also used for making predictions using the trained model.

A confidence threshold of 0.22 was set for object detection. If the model's confidence was below the detection threshold, a hoof was considered "unlabeled." Accuracy (Thrusfield, 1995) and Cohen's kappa were used to measure agreement between the model's binary outcome and a trained investigator. Because the M2 stage is infectious, statistical analysis was set to focus on the model's ability to detect M2 lesions. Both $\mathrm{M} 0 / \mathrm{M} 4 \mathrm{H}$ and unlabeled hooves were considered "not M2 lesions" when calculating Cohen's kappa.

For each DD image classification (M2 and M0/ $\mathrm{M} 4 \mathrm{H}$ ), the image database was randomly split into a training set $(70 \%)$ and a validation set $(30 \%)$. The moving average loss on the training set reached approximately 1.1 after 42,875 steps, and the moving average loss on the validation set reached approxi- 




Figure 1. Moving average of training and validation loss throughout training of the YOLOv2 model. The type of loss (training or validation) is indicated by the grayscale intensity as shown in the legend.

mately 1.4 (Figure 1). Individual M0, M4H, and M2 images were processed by the trained YOLOv2 model; Figure 2 illustrates the resulting lesion classifications and bounding boxes. Upon completion of training, the model achieved a validation set accuracy of $86 \%$ (444/516) for M0/M4H, and 56\% (296/529) for M2 lesions (Table 1). Combined accuracy for both image categories was $71 \%$ (740/1045). Using the library "irr" in $\mathrm{R}$ version 3.5.1 (cran.r-project.org), the model's overall agreement with human annotations was described by a Cohen's kappa of 0.51 .

Table 1. Performance of the YOLOv2 model

\begin{tabular}{lccc}
\hline Item & M0/M4H & M2 & Combined $^{3}$ \\
\hline Internal validation & & & \\
Correct, \% (n) & $86.0(444)$ & $55.9(296)$ & $70.8(740)$ \\
Incorrect, \% (n) & $4.5(23)$ & $5.9(31)$ & $5.2(54)$ \\
Unlabeled, \% (n) & $9.5(49)$ & $38.2(202)$ & $24.0(251)$ \\
$\quad$ Total (n) & 516 & 529 & 1,045 \\
External validation & & & \\
Correct, \% (n) & $89.2(846)$ & $59.0(20)$ & $88.2(866)$ \\
Incorrect, \% (n) & $5.3(50)$ & $38.0(13)$ & $6.4(63)$ \\
Unlabeled, \% (n) & $5.5(52)$ & $3.0(1)$ & $5.4(53)$ \\
Total (n) & 948 & 34 & 982 \\
\hline
\end{tabular}

${ }^{1}$ Noninfectious M-stages of digital dermatitis (the M-stage is a scoring system that classifies cows showing signs of digital dermatitis).

${ }^{2}$ An infectious M-stage of digital dermatitis.

${ }^{3}$ Total number of hooves analyzed from the M0/M4H and M2 categories.

${ }^{4}$ The YOLOv2 model did not provide a label, but the human evaluator did.
An external validation trial was performed in a rotary parlor on a commercial dairy farm. The purpose of the external validation was to classify unknown hooves in real time using a Logitech c930e webcam (Lausanne, Switzerland). On the farm, 491 cows were selected at random during milking on a rotating carousel and webcam videos of their feet were processed with the trained YOLOv2 model to identify DD lesion in real time. The cows' feet were washed with water before passing the camera, which is an element of the farm's usual milking routine. We also obtained video recording of the YOLOv2 output of the hooves passing the webcam with a clear bounding box encompassing the classified lesions. Predicted DD scores were recorded in a spreadsheet along with scores from the trained investigator. In total, 982 hooves from 491 cows were identified and scored as M0, M2, or $\mathrm{M} 4 \mathrm{H}$ by the trained investigator. The model achieved $89 \%(846 / 948)$ accuracy for M0/M4H lesions and 59\% (20/34) accuracy for M2 lesions. Combined accuracy for both image categories was 88\% (866/982). These results are depicted in Table 1. Cohen's kappa was calculated to be 0.36 . With respect to the position of cows' feet, the model needed image clarity and camera distance similar to the training image data set to detect DD lesions in real time.

Considering the accuracy and agreement measured by Cohen's kappa mentioned above, the YOLOv2 approach was applicable for DD detection in a milking parlor. Adapting the current model to use YOLOv3 TensorFlow version 2.0 and updated CUDA drivers 


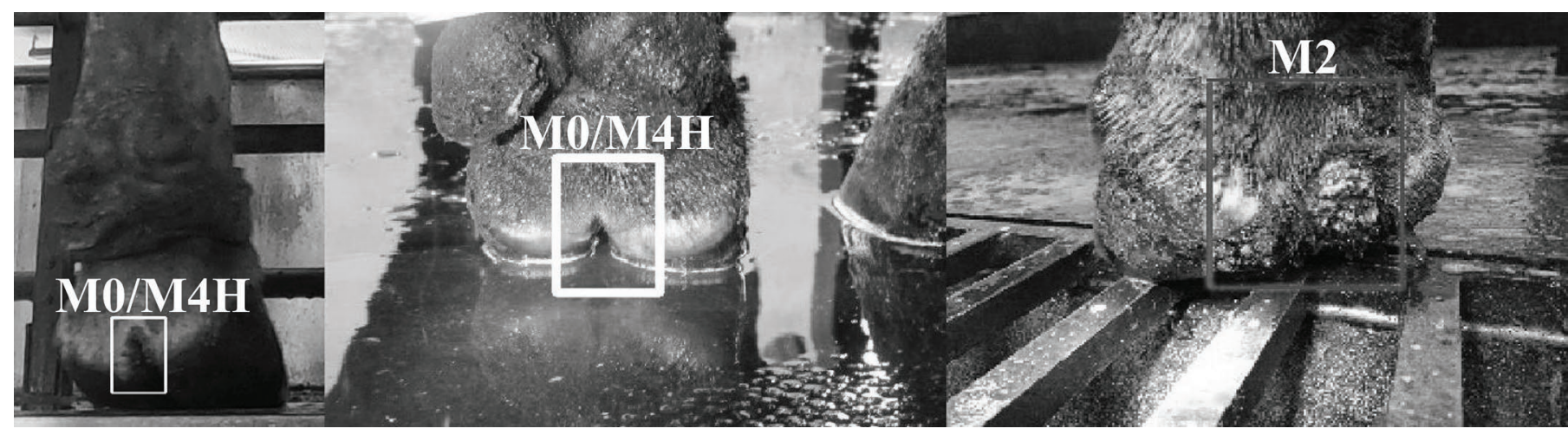

Figure 2. Left to right: properly labeled M4H, M0, and M2 digital dermatitis lesion photographs generated by the YOLOv2 model. M0/ $\mathrm{MH} 4=$ healthy claw/chronically affected claw; M2 = active ulcerative lesions.

would likely improve performance (Redmon and Farhadi, 2018). Moreover, it is important for future studies to compare other available COMV model architectures and identify which approaches work best in various application domains. For example, programs that use segmentation, such as Mask R-CNN (He et al., 2017), provide clearer detection boundaries and would better serve situations where hooves overlap, such as a heifer raising facility or feedlot. Nevertheless, in applications where hooves are clearly separated from one another, such as a milking parlor, YOLOv2 is an excellent approach for fast and clear classification of lesions.

The value of the combined external validation accuracy was greater than the combined accuracy achieved on the internal validation set. However, within the image categories of the internal and external validation, prediction accuracies were comparable. The model misclassified M2 lesions more often than M0/ $\mathrm{M} 4 \mathrm{H}$ lesions, which was due to a lower prevalence of M2 lesions (3.5\%) compared with the M0/M4H lesion prevalence $(96.5 \%)$ on the farm. This suggests that combined accuracy provides a misleading measure of general performance. Cohen's kappa provided a more appropriate measure of accuracy, and it was lower during the external validation. The M2 lesion detection accuracy is expected to increase with a higher M2 prevalence population. The estimated DD prevalence of endemic farms is highly variable, ranging from 3 to $30 \%$, according to the 2014 National Animal Health Monitoring System (NAHMS) report (United States Department of Agriculture, 2014). The performance degradation was expected because of the differences in image settings for each validation set. The internal validation images were randomly selected from the training database, and the external validation images were randomly selected from webcam footage at a single farm location. Scoring DD lesions in a labora- tory room versus on-farm contributes to the differences in accuracy between the internal and external validation. This suggests that performance may be improved with a more representative image training data set that mirrors what the YOLOv2 model is expected to interpret in external validation. Specifically, incorporating more webcam-derived images to the image training data set and implementing the model on more commercial dairy farms would improve overall performance.

In addition, unbalanced data sets are a well-known issue in COMV modeling (Chatterjee, 2018), and the M2 database (1,050 images) had fewer images than the $\mathrm{M} 0 / \mathrm{M} 4 \mathrm{H}$ database (2,591 images). Simply adding more images to the underrepresented database should improve the model. Distance discrepancy between the camera and the object is another known issue that can cause detection problems with YOLOv2 models (Redmon and Farhadi, 2018). A large portion of the DD images were screenshots from MP4 recordings of cows' feet, and consequently, the perspective was closer to the feet than what a camera in a real-world application would view. This distance discrepancy may explain the lower than expected accuracy; the addition of images to the training database from various distances is a potential solution.

Looking ahead, an ideal COMV model for DD detection would have 2 additional components: expansion of the training database to include more images and an increase the number of farms for greater on-farm implementation. Hoof diseases are present in many different types of production animal settings (Aguiar et al., 2011), and expanding the database to include the M4P DD M-stage, reverse corkscrew claws, white line disease, foot rot, and other hoof diseases would greatly advance the model's utility. The YOLOv2 framework allows for classifying additional diseases, and with the 
inclusion of sufficient hoof images, future studies may improve the disease detection abilities of the YOLOv2 model. The most robust prediction models will also require a training database that adequately represents different cattle breeds, animal species, and geographical regions.

Encouragement of greater on-farm use will also be essential for the development of COMV technology in the dairy industry. One way to increase implementation is generating an iOS or Android application to process images or videos using a trained YOLOv2 model. Using Docker, Flask, and other cloud-based containers, a model can be made available in handheld devices (Okoh, 2019). An application for handheld devices would allow commercial producers, veterinarians, hoof trimmers, and other cow professionals to take pictures of DD lesions and obtain scores of M-stages automatically in real time. This would also support a more rapid model development cycle by creating more opportunities for external validation and a richer supply of training images. In addition to the milking parlor, continuous DD monitoring could be implemented by generating foot images in cow traffic areas, milking robots, or cattle processing facilities to process images or videos with the COMV model. Predictions could be aggregated to describe herd-level disease trends, which would assist in DD prevention and treatment.

In conclusion, the YOLOv2 DD detection model developed in this study was able to identify and classify DD lesions on a commercial dairy farm with a fair degree of accuracy. Although implementing a YOLOv2 DD detection model is a step forward in bringing COMV to the dairy farm, this is merely a foundation to be built upon. Optimization of DD detection for treatment and expansion of the approach to include more diseases, even beyond those affecting hooves, has the potential to improve animal welfare at a pace previously unattainable.

\section{ACKNOWLEDGMENTS}

The authors gratefully acknowledge the Foundation for Food and Agriculture Research (Washington, DC) for the financial support for this research. The authors thank Jamie Sullivan (Carman, MB, Canada) and Chris Bauer (Mondovi, WI) for contributing images of hoof lesions. The authors have no stated conflicts of interest.

\section{REFERENCES}

Aguiar, G. M. N., S. V. D. Simões, T. R. Silva, A. C. O. Assis, J. M. A. Medeiros, F. Garino Jr., and F. Riet-Correa. 2011. Foot rot and other foot diseases of goat and sheep in the semiarid region of northeastern Brazil. Pesqui. Vet. Bras. 31:879-884. https://doi .org/10.1590/S0100-736X2011001000008.

Berry, S.L., D.H. Read, T.R. Famula, A. Mongini, and D. Döpfer. 2012. Long-term observations on the dynamics of bovine digital dermatitis lesions on a California dairy after topical treatment with lincomycin HCl. Vet. J. 193:654-658. https://doi.org/10 $.1016 /$ j.tvjl.2012.06.048.

Chatterjee, S. 2018. Deep Learning Unbalanced Training Data? Solve It like This. Accessed August 7, 2019. https://towardsdatascience .com/deep-learning-unbalanced-training-data-solve-it-like-this $-6 \mathrm{c} 528 \mathrm{e} 9 \mathrm{efea} 6$.

Chollet, F. 2016. Building Powerful Image Classification Models Using Very Little Data. Accessed March 17, 2020. https://blog .keras.io/building-powerful-image-classification-models-using -very-little-data.html.

Chollet, F. 2018. Deep Learning with Python. Manning Publications Co, Shelter Island, New Jersey.

Cramer, G., K. D. Lissemore, C. L. Guard, K. E. Leslie, and D. F. Kelton. 2009. Herd-level risk factors for seven different foot lesions in Ontario Holstein cattle housed in tie stalls or free stalls. J. Dairy Sci. 92:1404-1411. https://doi.org/10.3168/jds.2008 $-1134$.

Döpfer, D. 2009. Digital Dermatitis: The Dynamics of Digital Dermatitis in Dairy Cattle and the Manageable State of Disease. Accessed August 7, 2019. http://www.hoofhealth.ca/Dopfer.pdf.

Döpfer, D., A. A. H. M. ter Huurne, J. L. Cornelisse, A. J. A. M. van Asten, A. Koopmans, F. A. Meijer, Y. H. Schukken, I. Szakall, W. Klee, and R. B. Bosma. 1997. Histological and bacteriological evaluation of digital dermatitis in cattle, with special reference to spirochaetes and Campylobacter faecalis. Vet. Rec. 140:620-623. https://doi.org/10.1136/vr.140.24.620.

Gutierre, D. 2018. Overview of the YOLO Object Detection Algorithm. Accessed August 8, 2019. https://opendatascience.com/ overview-of-the-yolo-object-detection-algorithm.

He, K., G. Gkioxari, P. Dollár, and R. Girshick. 2017. Mask R-CNN. arXiv: 1703.06870 .

Jacobs, C., C. Beninger, G. S. Hazlewood, K. Orsel, and H. W. Barkema. 2019. Effect of footbath protocols for prevention and treatment of digital dermatitis in dairy cattle: A systematic review and network meta-analysis. Prev. Vet. Med. 164:56-71. https://doi.org/10.1016/j.prevetmed.2019.01.011.

Krull, A. C., J. K. Shearer, P. J. Gorden, H. M. Scott, and P. J. Plummer. 2016. Digital dermatitis: Natural lesion progression and regression in Holstein dairy cattle over 3 years. J. Dairy Sci. 99:3718-3731. https://doi.org/10.3168/jds.2015-10535.

Moore, S. 2012. Employee Turnover on Dairy Farms and What It's Costing Producers. Accessed August 7, 2019. https://www.canr .msu.edu/news/employee_turnover_on_dairy_farms_and_what _its_costing_producers.

Okoh, M. 2019. How to Build and Deploy a Flask Application Using Docker on Ubuntu 18.04. Accessed August 7, 2019. https://www .digitalocean.com/community/tutorials/how-to-build-and-deploy -a-flask-application-using-docker-on-ubuntu-18-04.

Redmon, J. 2013. Darknet: Open Source Neural Networks in C. Accessed August 15, 2019 https://pjreddie.com/darknet/.

Redmon, J., S. Divvala, R. Girshick, and A. Farhadi. 2015. You Only Look Once: Unified, Real-Time Object Detection. arXiv: 1506.02640 .

Redmon, J., and A. Farhadi. 2018. YOLOv3: An Incremental Improvement. arXiv:1804.02767.

Sandle, T. 2018. Facial Recognition Is Set to Disrupt Agriculture. Accessed August 7, 2019. http://www.digitaljournal.com/news/ environment/facial-recognition-is-set-to-disrupt-agriculture/ article/528320.

Schulz, T., Y. Gundelach, M. Feldmann, and M. Hoedemaker. 2016. Early detection and treatment of lame cows. Tierarztl. Prax. Ausg. G Grosstiere Nutztiere 44:5-11. https://doi.org/10.15653/ TPG-150534.

Thrusfield, M. 1995. Veterinary Epidemiology, 2nd ed. Blackwell Science Ltd., Oxford, UK. 
United States Department of Agriculture. 2014. Dairy 2014 Health and Management Practices on U.S. Dairy Operations, 2014. Accessed March 17, 2020. https://www.aphis.usda.gov/animal _health/nahms/dairy/downloads/dairy14/Dairy14_dr_PartIII .pdf.

Vanhoudt, A., D. A. Yang, T. Armstrong, J. N. Huxley, R. A. Laven, A. D. Manning, R. F. Newsome, M. Nielen, T. van Werven, and N. J. Bell. 2019. Interobserver agreement of digital dermatitis Mscores for photographs of the hind feet of standing dairy cattle. J. Dairy Sci. 102:5466-5474. https://doi.org/10.3168/jds.2018 -15644 .
Viera, A. J., and J. M. Garrett. 2005. Understanding interobserver agreement: The kappa statistic. Fam. Med. 37:360-363.

Zinpro Inc. 2019. Digital Dermatitis in Beef and Dairy Cattle. Accessed August 7, 2019. https://www.zinpro.com/lameness/digital -dermatitis.

\section{ORCIDS}

Preston Cernek @ https://orcid.org/0000-0002-5276-7284 Nathan Bollig @ https://orcid.org/0000-0002-0393-6395 